\title{
Ketofol: is it the best sedoanalgesic for pediatric procedures outside the operating room?
}

\author{
Eduardo Mekitarian Filho ${ }^{\text {* }}$ (1)
}

To the Editor,

It was with great interest that I read the article by Hayes JA et al. ${ }^{1}$, entitled "Safety and efficacy of the combination of propofol and ketamine for procedural sedation/anesthesia in the pediatric population: a systematic review and meta-analysis" in the present issue of Anesthesia \& Analgesia.

In fact, the need of pediatric procedures has increased in the past years, due to more availability and knowledge of adverse events from sedatives and other diseases. It would obviously be presumed that the emergency pediatrician had to be trained well to perform safe sedation, since an anesthesiologist cannot be free all the time. After the "death" of chloral hydrate, this situation required major concerns. Propofol, a high extremely effective hypnotic agent, with or without the sedative midazolam, had been used in place of other potential gravity drugs. Ketamine, a unique sedative and analgesic, also has been used safely in fast procedures that require sedation and analgesia. The combination of these two drugs, also referred to as "ketofol," has gained many advantages in reducing the doses and adverse events of one of them alone. Propofol is a very short-acting agent that provides no analgesia. It can cause adverse events such as hypotension and bradycardia if large doses are administered, and its use is prohibited by the Food and Drug Administration (FDA) for intensive care units (ICU) for more than $24 \mathrm{~h}$, although several articles reported a much longer time with no side effects ${ }^{2}$. Ketamine, an N-methyl-D-aspartate acid (NMDA) receptor antagonist, produces a dissociated state with minimal risk of airway compromise or apnea if administered slowly, and stable or elevated hemodynamic (blood pressure and heart rate) parameters, antagonizing possible effects of propofol, but may cause nausea, vomiting, and delirium during the recovery phase, which is very rare.

El Mourad et al. ${ }^{3}$ studied the effects of ketofol, propofol, and dexmedetomidine (DEX) for transesophageal echocardiography (TEE). The time onset and offset of sedation, duration of TEE procedure, and the need for rescue propofol were significantly less in the propofol and ketamine groups compared with the group using DEX. Rao et al. ${ }^{4}$ found that, compared with placebo, midazolam, and opioids, DEX significantly decreased the incidence of post-anesthesia emergency agitation or delirium in pediatric patients. However, DEX did not exhibit this superiority compared with propofol and ketamine. The significant difference was not exhibited compared with propofol (or pentobarbital) [OR (odds ratio) $0.56,95 \% \mathrm{CI}$ (confidence interval) 0.15-2.14, $\mathrm{p}=0.39$ ], ketamine [OR 0.43, 95\%CI 0.19-1.00, $\mathrm{p}=0.05$ ], clonidine [OR $0.54,95 \%$ CI $0.20-1.45, \mathrm{p}=0.22$ ], chloral hydrate [OR 0.98, 95\%CI 0.26-3.78, $\mathrm{p}=0.98$ ], melatonin [OR 1.0, 95\%CI 0.13-7.72, p=1.00], and ketofol [OR 0.55, 95\%CI 0.16-1.93, $\mathrm{p}=0.35$ ].

Rayes et al. ${ }^{1}$ found that other combination of drugs (e.g., chloral hydrate and fentanyl), compared with ketofol (administered either together or in separate bolus), had no statistical difference in providing tachycardia (OR 1.27; 95\%CI 0.891.8) but was safer in the bradycardia group, with a tendency of protection (OR 0.61, 95\%CI 0.38-0.98). Light hypotension without the need for intervention was also more associated with ketofol (OR 0.52; 95\%CI 0.37-0.73). Because of these reasons, it is important to administer ketofol for painful and sedative-requiring procedures.

Stevic et al. ${ }^{4}$ studied 203 patients aged one month to 15 years who received sedation for laser therapy, using either combined ketamine and fentanyl or ketofol alone. Tachycardia was recorded in a significantly higher number of patients who received ketamine as the anesthetic agent (35.9 versus 3\%). Hypertension was also significantly more frequent in patients who received ketamine in comparison with patients who received ketofol (25.2 versus 3\%). Laryngospasm, a dangerous but rare adverse event for ketamine, was not observed in both the examined groups. There was no statistically significant difference between groups in satisfaction of parents and doctors.

${ }^{1}$ Hospital Santa Catarina - São Paulo (SP), Brazil.

*Corresponding author: emf2002@uol.com.br

Conflicts of interest: the authors declare there are no conflicts of interest. Funding: none.

Received on April 27, 2021. Accepted on May 30, 2021. 
Apnea and respiratory depression occurred significantly more frequent in ketofol than in ketamine group (12 versus $0.97 \%$ and 13 versus $0 \%$ ).

Gulec et al. ${ }^{5}$ randomized 60 children for elective circumcision and compared the effects of a mixture of ketamine alone or ketofol. A difference in the initial pulse rate was observed ( $p>0.050$ ). Initial diastolic blood pressure and subsequent serial measurements of 5, 10, 15, and 20 minutes; systolic blood pressure; diastolic blood pressure; and pulse rate in ketamine group were significantly higher $(\mathrm{p}<0.050)$. A difference of the initial pulse rate was observed $(p>0.050)$. Initial diastolic blood pressure and subsequent serial measurements of 5, 10, 15, and 20 minutes; systolic blood pressure; diastolic blood pressure; and pulse rate in ketamine group were significantly higher $(\mathrm{p}<0.050)$. They concluded that ketofol provides better sedation quality and hemodynamic than that provided by ketamine alone in pediatric circumcision operations. The authors did not observe significant complications during sedation in these two groups. Therefore, ketofol appears to be an effective and safe sedation method for circumcision operation.

In conclusion, even with a few articles mentioned in this study, ketofol has a short pathway to become the best pediatric procedure sedative outside the operating room, with data from Hayes et al. ${ }^{1}$ being confirmed by other good-quality articles. This article is extremely well-written and clear and concludes that using propofol with ketamine is safe, since you have in your hands appropriate resuscitation tools and are prepared to conduct the side effects, albeit rare, when you need to do this.

\section{REFERENCES}

1. Hayes JA, Aljuhani T, De Oliveira K, Johnston BC. Safety and efficacy of the combination of propofol and ketamine for procedural sedation/anesthesia in the pediatric population: a systematic review and meta-analysis. Anesth Analg. 2021;132(4):979-92. https://doi.org/10.1213/ ANE.0000000000004967

2. Abola R, Geralemou S, Szafran M, Gan TJ. Intravenous anesthetics. In: Barash PG, Cullen BF, Stoelting RK, Cahalan MK, Stock MC, editors. Clinical anesthesia. 7th ed. Philadelphia: Lippincott Williams \& Wilkins; 2017. p.493-6.

3. Hayes JA, Aljuhani T, De Oliveira K, Johnston BC. Safety and efficacy of the combination of propofol and ketamine for procedural sedation/anesthesia in the pediatric population: a systematic review and meta-analysis. Anesth Analg. 2021;132(4):979-92. https://doi.org/10.1213/ ANE.0000000000004967

4. Rao $Y$, Zeng $R$, Jiang $X$, Li J, Wang $X$. The Effect of dexmedetomidine on emergence agitation or delirium in children after anesthesia-a systematic review and metaanalysis of clinical studies. Front Pediatr. 2020;8:329. https:// doi.org/10.3389/fped.2020.00329

5. Stevic M, Ristic N, Budic I, Ladjevic N, Trifunovic B, Rakic I, et al. Comparison of ketamine and ketofol for deep sedation and analgesia in children undergoing laser procedure. Lasers Med Sci. 2017;32(7):1525-33. https://doi.org/10.1007/s10103017-2275-x 